\title{
Listening comprehension across the adult lifespan
}

\author{
Mitchell S. Sommers, Ph.D., \\ Department of Psychology, Washington University \\ Sandra Hale, Ph.D., \\ Department of Psychology, Washington University \\ Joel Myerson, Ph.D., \\ Department of Psychology, Washington University \\ Nathan Rose, Ph.D., \\ Department of Psychology, Washington University
}

Nancy Tye-Murray, Ph.D., and Department of Otolaryngology, Washington University School of Medicine

Brent Spehar, Ph.D.

Department of Otolaryngology, Washington University School of Medicine

\section{Short Summary}

The current study provides the first systematic assessment of listening comprehension across the adult lifespan. A total of 433 participants ranging in age from 20-90 listened to spoken passages and answered comprehension questions following each passage. In addition, measures of auditory sensitivity were obtained from all participants to determine if hearing loss and listening comprehension changed similarly across the adult lifespan. As expected, auditory sensitivity declined from age 20 to age 90 . In contrast, listening comprehension remained relatively unchanged until approximately age 65-70, with declines evident only for the oldest participants.

\begin{abstract}
Most previous investigations of age-related changes in the perception of spoken language have employed extreme-groups designs in which the performance of a group of old adults (typically individuals over age 65) is compared to that of a group of younger adults (typically individuals below age 25). In addition, a majority of these investigations have focused on age-related changes in the perception and identification of relatively brief spoken materials, including isolated phonemes, individual words, and short sentences (Nittrouer \& Boothroyd, 1990; Humes, 1996; Sommers \& Danielson, 1999). The results of these studies provide clear evidence that speech perception differs for groups of young adults and older adults and that age-related hearing loss is a significant contributor to the differences between young and older listeners (CHABA, 1988; Humes, 1996).

In contrast to these earlier studies, the current work focuses on how age affects the ability to understand extended spoken passages and uses questions that assess comprehension of the material rather than identification of individual elements. In addition, we have adopted a cross-sectional approach to assessing changes in listening comprehension across the adult lifespan. One of the important advantages of the cross-sectional approach is that it provides a methodology for determining whether the well-documented changes in auditory sensitivity across the adult lifespan produce parallel changes in listening comprehension. Importantly,
\end{abstract}

Correspondence related to this work should be sent to Mitchell Sommers, Department of Psychology, Washington University, 1 Brookings Drive, Campus Box 1125, 63130. msommers@wustl.edu. 
changes in auditory sensitivity can begin as early as the third decade of life and accelerate across the lifespan (Morrell et al., 1996). Evidence for similar functions relating auditory sensitivity and listening comprehension to age would suggest that age-related hearing loss not only contributes to differences in speech perception between older and younger adults, but also accounts for the age-related decline in older adults' ability to comprehend the meaning of spoken passages.

Consistent with the proposal that auditory sensitivity and listening comprehension follow similar trajectories across the adult lifespan, Schneider et al. (2000) used an extreme-groups design to investigate whether listening comprehension differed between young (mean age approximately 23) and older (mean age approximately 70) adults. In a series of experiments, groups of young and older participants listened to extended passages (10-15 min.) and after each passage participants were asked a series of detail and integrative multiple-choice questions. When young and older adults were tested under identical listening conditions, even older adults with relatively mild hearing losses $(<25 \mathrm{~dB}$ HL for $2 \mathrm{kHz}$ and below; < 35 $\mathrm{dB} \mathrm{HL}$ for $4 \mathrm{kHz}$ ) had significantly poorer listening comprehension than young adults with normal auditory sensitivity. When audibility was equated across groups, however, either by using older listeners with minimal hearing loss or by adjusting the signal-to-noise ratio on an individual basis, young and older adult groups showed nearly identical listening comprehension. These results suggest that even small declines in auditory sensitivity can impair the ability to understand spoken passages. Of particular importance to the lifespan perspective adopted in the current investigation, age-related hearing loss can begin as early as the third decade of life and the decline accelerates across the lifespan (Morrell, et al., 1996). Thus, the findings from Schneider et al. would predict relatively similar functions relating hearing loss and listening comprehension across the adult lifespan.

A second reason that increasing age might be associated with impaired listening comprehension is that even if older adults are able to achieve adequate speech recognition, the increased perceptual effort needed to overcome age-related auditory dysfunction may impose a "down-stream" cost on cognitive abilities that are critical for message comprehension. McCoy et al. (2005), for example, compared memory for short sequences of words in two groups of older adults who varied in their degree of hearing loss. Listeners were presented with single words and were asked periodically to recall the last three words that had been presented. Both groups were able to identify the words correctly, as evidenced by similar recall performance for the final word in the memory tests. The hearing-impaired listeners, however, exhibited significantly reduced recall of words presented early in the sequence compared to their better-hearing counterparts. McCoy et al. attributed this reduced memory performance to downstream effects of reduced auditory sensitivity. In particular, they suggested that the hearing-impaired older adults obtained lower fidelity auditory information as a result of their hearing loss and thus had to expend greater perceptual effort to encode the signals. One consequence of this increase in initial processing demands is that subsequent memory performance was impaired relative to the better-hearing group. To the extent that listening comprehension depends on the ability to remember previously presented material and then integrate it with new information, the results of McCoy et al. (2005) would again predict a similar relationship between auditory sensitivity and listening comprehension across the adult lifespan.

To our knowledge, only one previous investigation (Kaufman \& Horn, 1996) has examined changes in the ability to understand extended spoken passages across the adult lifespan (see, however, Obler et al., 1985 for evidence of age differences in the ability to answer yes-no questions based on auditory presentations of short paragraphs). Kaufman and Horn (1996) tested a normative sample of adults between the ages of 17 and 94 on the eight subtests of the Kaufman Adolescent and Adult Intelligence Test. For the Auditory Comprehension 
subtest, participants heard recordings of a news story and then answered both detail-specific and inferential questions about the content. Performance declined moderately from the youngest age group through middle adulthood (until about age 55), followed by an accelerated decline during late adulthood. An unfortunate limitation of the Kaufman and Horn data is that because auditory acuity was not assessed it is not possible to determine the extent to which changes in listening comprehension paralleled any changes in hearing loss.

The present investigation was designed to provide a systematic evaluation of how the ability to understand spoken passages changes across the adult lifespan and how listening comprehension is related to auditory sensitivity. A large $(n=433)$ cross-sectional sample of adults, whose ages ranged from 20-89 and who had age-appropriate hearing ability, completed several measures of listening comprehension and auditory sensitivity. Of interest was whether hearing loss and listening comprehension follow similar trajectories across the adult lifespan and whether age-related differences in comprehension can be entirely accounted for by differences in auditory sensitivity.

\section{Method}

\section{Participants}

A total of 433 participants, ranging in age from 20 to 89 , were recruited to take part in the study. Participants were selected such that we would have approximately 60 (range $=58-66$ ) individuals in each of 7 age decades (20-29, 30-39, 40-49, 50-59, 60-69, 70-79 and 80-89). None of the participants reported wearing hearing aids or other assistive listening devices, and all were native speakers of English. To minimize the likelihood of including individuals with cognitive impairment, participants were administered the Telephone Interview for Cognitive Status (TICS; Brandt et al., 1988) during the initial recruitment contact and only individuals who scored above 26, comparable to a score of 28 on the Mini-Mental State Examination (de Jager, Budge, \& Clarke, 2003), were invited to participate. All participants also received the Vocabulary subtest of the Wechsler Adult Intelligence Scale (WAIS III: Wechsler, 1997); raw vocabulary score and age were not correlated, $r=-.06, p>.10$.

\section{Audiometric thresholds}

A principal consideration in the current study was to select participants with hearing thresholds typical for their age. Morrell et al. (1996) reported normative hearing ranges for octave frequencies from $250-4000 \mathrm{~Hz}$ in individuals between 20 and 90 years old who had been screened for otological disorders and noise-induced hearing loss. All participants in the current study had hearing thresholds within the ranges established by Morrell et al. Figure 1 presents the mean hearing loss in the best ear as a function of frequency for participants in each of the 7 decades studied. As expected, participants exhibited a systematic and progressive high-frequency hearing loss as a function of age.

\section{Listening comprehension measures}

To assess listening comprehension across a broad range of material that varied in length and topic, we included stimulus materials from two existing comprehension measures: short passages and questions from the Scholastic Aptitude Test (SAT; Claman, 1997) and a longer passage and questions from the Brown, Carlsen, Carstens listening comprehension test (BCC; Brown et al., 1995). In addition, we used passages and questions from a measure of listening comprehension recently developed in our laboratory, the Lectures, Interviews and Spoken Narratives (LISN) test (Tye-Murray et al., 2008).

SAT-Five passages were selected from a commercially available SAT preparation book (Claman, 1997) and were recorded by a female speaker. Passages were approximately 2-3 
minutes in length, and there were four questions with four response alternatives for each passage.

BCC-The BCC test (Brown et al., 1995) is one of the few comprehension tests specifically designed to assess listening rather than reading comprehension. The $\mathrm{BCC}$ involves listening to an extended passage (approximately 10 minutes) and then answering 21 five-alternative, multiple-choice questions designed to assess the ability to remember details, get central ideas, draw inferences, understand organization, and distinguish relevant from irrelevant information. The same female speaker used to record the SAT passages also recorded the BCC passage.

LISN-We developed the LISN (Tye-Murray et al., 2008) as another measure designed specifically to assess listening comprehension. The LISN contains three different types of passages: Lectures, Interviews, and Spoken Narratives. The lecture passages were selected from the BBC Reith lectures, a series of radio lectures by leading world figures discussing issues of contemporary interest. Interview passages were from the Booknotes series on CSPAN in which contemporary non-fiction authors discussed their books, their research, and their lives. The spoken narrative passages were selected from personal descriptions of life experiences collected in the Rutgers University Oral History Archives. Because all three types of comprehension passages were taken from transcripts of actual extended utterances in situations representative of those encountered by listeners in everyday life, they should have relatively high ecological validity.

The version of the LISN used in the current study consisted of six passages, two each of the lectures, interviews and spoken narratives. Each passage was approximately 3-5 minutes in duration. Six professional actors ( 3 males and 3 females) made audio and video recordings of the passages (only audio recordings were used in the current study). For each passage, we developed questions designed to assess different aspects of listening comprehension.

Information questions asked participants to recall a specific piece of information, whereas integration questions were designed to assess individuals' ability to combine two or more separate pieces of information. Finally, inference questions measured the ability to derive implications from the information presented in the passage. Two questions of each type were developed for each passage, yielding six questions for each of the six passages and thus a total of 36 questions for the entire test. Questions were multiple-choice format with 4 possible response alternatives for each question.

Two sets of pilot studies were conducted to refine the original set of comprehension questions for the LISN. First, to minimize the effects of differences in general knowledge across participants we conducted a series of pilot experiments in which young and older participants were asked to answer the questions without reading the passages. The questions and alternatives were modified iteratively until performance (without reading the passage) was approximately at chance ( $25 \%$ correct) for all questions.

A second pilot study was conducted to determine the appropriateness of the question designations as assessing information, integration and inference. We recruited 15 graduate students from the Washington University English Department to read the passages and categorize the questions into one of the three types. Mean overall agreement between our original categorization and that of the graduate students' for the three question types was 94\% (information), 85\% (integration), and 81\% (inference). Discrepancies resulted primarily from putatively inference questions being classified as integration questions and vice versa, and may simply reflect differences in emphasis, as answering the inference questions often required some integration of the presented material. 
Cronbach's alpha was computed separately for each age group to assess the internal consistency of the final version of the LISN and these values were moderate to high across the age groups, with all values exceeding .70. These results indicate not only that internal consistency was good overall, but also that the reliability of the measure was similar across the adult lifespan.

\section{Recordings}

For all three tests, speakers first read through the passages they were to produce prior to recording in order to familiarize themselves with the materials. During the recording, the text was displayed on a teleprompter and speakers were instructed to read the material in a "natural" or "conversational" style. Audio recordings were converted to digital files (16-bit, $44.1 \mathrm{kHz}$ sampling rate). After recording, all passages were amplitude-normalized to minimize level differences between the recordings.

\section{Procedure}

Participants heard the passages presented over a loudspeaker in a sound-attenuating test booth at a comfortable listening level (approximately $75 \mathrm{~dB}$ SPL). Following each passage, participants read the comprehension questions and response alternatives on a 17-in computer monitor and responded by pressing one of four response buttons. No feedback was provided. All participants heard the passages in the same order for the LISN (lecture, interview, narrative, lecture, interview, narrative) and the SAT (the BCC had only a single passage). For all three comprehension measures, the order of questions was randomized once for each passage and this same order was used for all participants.

\section{Results}

Scores on the three listening comprehension tests were strongly correlated, with $r$ s ranging from .62 to .67 . The mean percent correct for males and females were highly similar on all three tests, with differences between average male and female scores of $0.3 \%$ or less. To confirm that there were no sex $\times$ age interactions, we conducted analyses using the General Linear Model (GLM) for each comprehension test. As expected, there was a main effect of age for each test, all $F$ values $>5.40, p \mathrm{~s}<.05$, and no main effects of sex, all $F \mathrm{~s}<1.0$. Importantly, there were no sex $\times$ age interactions, all $F \mathrm{~s}<1.0$, and accordingly, all of the remaining analyses were conducted with performance collapsed across sex.

Mean percent correct on the LISN, SAT, and BBC for participants in each decade is depicted in the top panel of Figure 2. Visual inspection suggested that listening comprehension changes nonlinearly with age, and this was confirmed by polynomial regression: The quadratic coefficient was significant for each test: $t(430)=4.34,3.78$, and 2.44 for the LISN, SAT, and BCC, respectively, $p s<.015$. Because performance appeared to remain relatively constant up to a certain age and then decrease rapidly thereafter, we used piecewise linear regression to estimate the point at which the decline began. For all three tests, the breakpoints were between 65 and 70 years (see Table 1). The contrast between fitting a second-order polynomial and piecewise regression is illustrated in the bottom panel of Figure 2, which shows composite (mean z) listening comprehension scores as a function of age.

Based on the piecewise regression results, for each test we divided our participants into a younger group whose age was less than the breakpoint for that test and an older group whose age was equal to or greater than the breakpoint. For each test, there was a significant negative correlation between listening comprehension and age for those participants whose age was equal to or greater than the breakpoint; in contrast, listening comprehension and age 
were uncorrelated in those participants whose age was less than the breakpoint (see Table 1). Pairwise comparisons of the rates of decline after the breakpoint (in z-score units per year) on the LISN, SAT, and BCC tests failed to reveal any significant differences between the three listening comprehension tests: $F(1,144)=2.40$ for LISN vs. BCC, $p>.12$, and $F<1.0$ for both LISN vs. SAT and BCC vs. SAT. Performance on all three tests declined at a rate of about $0.05 \mathrm{z}$-score units per year. Although the differences in performance between participants of a particular age and those who were one year older were small, these differences accumulate, and the present results suggest that between 65 and 90 years of age, on average the total decline in listening comprehension will be more than one full z-score unit (see Figure 2).

Having demonstrated that listening comprehension declines with age in older adults, the next question was whether the observed decline was a consequence of the age-related decrease in auditory sensitivity (see Figure 1). To address this question, we computed the average of pure tone thresholds for both low (PTAL: 250, 500, $1000 \mathrm{~Hz}$ ) and high frequencies (PTAH: 2000, 4000, and $8000 \mathrm{~Hz}$ ) for each participant's best ear and then calculated the correlations between PTAL and PTAH and age. Both PTAL and PTAH were negatively correlated with age $(r=-.55$ and $r=-.50$, respectively, both $p s<.0001)$ in those participants over age 65 . These declines in auditory sensitivity may be seen in Figure 3 , which plots the pure tone thresholds at different frequencies as a function of age. Importantly for current purposes, the trajectories for the frequencies making up PTAH show declines beginning prior to the age-related decrease in listening comprehension; in contrast, the thresholds for the frequencies making up PTAL are relatively unchanged until after age 65 , and as was the case for listening comprehension, show substantial change only after this point.

For each of the three listening comprehension tests, we calculated the partial correlations between age and listening comprehension for those whose age was equal to or greater than the breakpoint, controlling for PTAL and PTAH. Despite the similarity in the zero-order correlations between PTAL and PTAH and age, controlling for PTAL resulted in a considerable reduction in the correlation between age and listening comprehension on all three comprehension tests, whereas controlling for PTAH had little or no effect. The partial correlations were statistically significant in all cases (see the two right-most columns in Table 1), indicating that advancing age was associated with a significant decrease in listening comprehension over and above that attributable to hearing loss.

As shown in Figure 2, hearing loss tends to accelerate for the oldest participants, especially for frequencies of $1000 \mathrm{~Hz}$ and above. In fact, the function relating age and hearing loss for frequencies above $500 \mathrm{~Hz}$ in the oldest group of participants (> 79) is similar to the function relating age and listening comprehension for these individuals. To investigate how hearing loss affects listening comprehension for these oldest participants, we computed partial correlations between age and listening comprehension controlling for PTAL and PTAH. Significant correlations between age and the composite measure of comprehension (in zscore units) remained significant $r=-.333, \mathrm{p}<.05$ controlling for PTAL and $\mathrm{r}=-.336, \mathrm{p}<$. 05 controlling for PTAH in this sub-group of our oldest participants. These findings reinforce the conclusion that under the very good listening conditions tested in the current study, auditory sensitivity accounts for some, but not all, of the age-related variance in the ability to understand spoken passage, even in the sample of participants with the steepest sloping audiograms.

Next, multiple regression was used to estimate exactly how much of the age-related decline in listening comprehension was attributable to hearing loss. For these analyses, we again focused on those participants whose age was equal to or greater than the breakpoint for each 
comprehension test. For each test, we first entered PTAL and PTAH separately into the regression model and then examined whether age accounted for additional variance in listening comprehension beyond that accounted for by hearing alone. Similar results were obtained for all three listening comprehension tests. PTAL accounted for a significant proportion of the variance but adding PTAH did not increase that proportion significantly, whereas age did contribute variance over and above the hearing variables. More specifically, PTAL, PTAH, and age together accounted for $16.7 \%$ of the variance in LISN scores, $23.6 \%$ of the variance in SAT scores, and $14.1 \%$ of the variance in BCC scores. For the LISN, age uniquely accounted for $7.2 \%$; for the $\mathrm{SAT}$ and $\mathrm{BCC}$, the corresponding percentages were $6.1 \%$ and $6.0 \%$.

It should be noted that the observed age-related decline in listening comprehension is not attributable to fatigue on the part of older adults. Although all three comprehension tests were administered on the same day, numerous rest breaks were provided. More importantly, the design of the LISN allowed us to address this concern by comparing performance on the first and second halves of the test because participants received exactly the same types of passages and questions in the first and second halves. Each half of the LISN contained one interview, one lecture, and one narrative, with two questions of each type for each of the three passages. For participants 65 and younger, performance on the second half of the LISN was slightly, but not significantly, better on the second half of the test. Importantly, the same was true for participants 66 and older, arguing against fatigue as an explanation for the decline in listening comprehension observed in the older adults.

\section{Comparisons across LISN passage types}

In order to determine whether the degree of age-related decline in listening comprehension depends on the type of extended speech, we compared the rates of age-related decline in comprehension of spoken interviews, lectures, and narratives, using passages of each type from the LISN. Performance on the three passage types differed significantly in the group of participants 66 and over, $F(2,290)=47.38, p<.001$, with the lectures being more difficult than either the interviews or narratives: percent correct $=48.3,61.2$, and 62.4 , respectively. Despite the difference in difficulty, the rates of age-related decline (measured in $\mathrm{z}$-score units per year) did not differ among passage types: $F(1,144)=1.11$ for interviews vs. narratives, $F(1,144)=2.93$ for lectures vs. narratives, and $F<1.0$ for lectures vs. interviews, all $p s>.09$.

\section{Comparison across LISN question types}

To determine if rates of age-related decline in listening comprehension differed for individuals 66 and over as a function of question type, we compared rates of decline for the information, integration, and inference questions in the LISN combined across the six passages. Overall performance on the three types of questions differed significantly $F(2$, $290)=91.4, p<.001$, with performance best on information questions $(M=69.2)$, intermediate on integration questions $(\mathrm{M}=55.4)$ and poorest on inference questions $(\mathrm{M}=$ 49.7). Despite the greater difficulty of the inference questions, the rate of age-related change did not differ as a function of question type, all $F s<1.1$, all $p s>.20$.

\section{Discussion}

The results of the present study provide the first detailed description of how comprehension of extended spoken passages varies across seven decades of the adult lifespan. Overall, listening comprehension remained relatively unchanged for most of this period, with declines starting between 65 and 70 and continuing thereafter. Not surprisingly, age-related decreases in auditory sensitivity, especially low-frequency PTA, accounted for a 
considerable portion of the observed decline in listening comprehension. In addition, however, analyses revealed that for participants over 65 , age was associated with a reduced ability to comprehend spoken passages, even after controlling for hearing loss. Finally, the decline in listening comprehension observed in the older participants was not the result of a particular difficulty with one type of spoken passage or one type of comprehension question. The three types of LISN passage (lectures, interviews, and narratives) and the three question types associated with each passage (information, integration and inference) differed in absolute levels of performance, but in all cases similar rates of age-related decline were observed.

It should be noted that participants in the current study were tested under nearly ideal listening conditions - stimuli were presented via headphones at relatively high signal-tonoise ratios in a sound-attenuating booth - which may have served to minimize some of the potential negative effects of hearing impairment. Although hearing loss is likely to play a larger role in more degraded auditory environments, the conditions in the current study were designed to permit a test of whether other age-related factors besides hearing (e.g., age differences in perceptual and cognitive abilities) play a significant role in listening comprehension, and the answer is clearly yes. It is left to future research to identify these factors, and to assess their relative contributions under varied listening conditions.

The use of speech intelligibility measures may be useful in this regard, as there is considerable evidence that age-related changes in pure tone thresholds underestimate reductions in intelligibility, especially under more challenging listening conditions (Rajan \& Cainer, 2008). For example, there is emerging evidence that presbycusic changes in the peripheral auditory system can impair suprathreshold auditory functions, including temporal and frequency resolution, thereby leading to decreased intelligibility (Schneider \& PichoraFuller, 2001). Because these impairments may be at least partially independent of hearing loss, it is possible that they may also have contributed to the reduced ability of the older participants in the current study to understand spoken passages.

One of the principal questions raised by the results of the current study is what might account for the pattern of results we observed, in which listening comprehension remains relatively unchanged well into the sixth decade of life, but declines substantially for individuals over age 65. If we use the performance of our youngest participants, who show minimal changes in auditory sensitivity, as a baseline, then we can first consider how our middle-aged adults (approximately age 40-65), with mild-to-moderate hearing losses, were able to maintain relatively consistent (compared with the youngest group) listening comprehension performance despite their reduced auditory sensitivity. The similarity in passage understanding for these two groups (young and middle-aged) is even more surprising in light of results from the few cross-sectional studies (Jerger, 1973; Gates et al., 1990; Cruickshanks et al., 1998) that have demonstrated declines in isolated word identification from middle aged to older adults (the youngest group tested was 48-59). We propose two possible, not mutually exclusive, explanations for the well-preserved listening comprehension in our middle-aged adults.

First, the nature of presbycusic hearing loss is such that for middle-aged individuals (approximately 40-65) significant reductions in auditory sensitivity are primarily confined to higher frequencies (i.e., $2 \mathrm{kHz}$ and greater), and lower frequencies are more important for the intelligibility of connected discourse as compared to nonsense syllables and isolated words (American National Standards Institute, 1997; Studebaker, Pavlovic, \& Sherbecoe, 1987). Findings from the multiple regression analyses in the current study also support the greater importance of low-, as opposed to high-frequency information in maintaining listening comprehension. As Hornsby and Ricketts (2006) pointed out, speech information is 
somewhat redundant across frequencies and many individuals with high-frequency losses are able to make good use of low-frequency information. This is especially true when speech stimuli are presented in quiet (e.g., Hogan \& Turner, 1998; Ching, Dillon, \& Byrne, 1998), and thus the fact that the spoken passages in the current study were presented in quiet may have contributed to the preservation of listening comprehension in middle-aged participants with primarily high-frequency hearing loss.

A second factor that may have contributed to the well-preserved listening comprehension abilities of individuals from age 40-65 is that the spoken materials used in the current study provide a rich semantic context that can compensate for age-related changes in both auditory sensitivity and word recognition (Hutchinson, 1989; Nittrouer \& Boothroyd, 1990; Sommers $\&$ Danielson, 1999). Specifically, previous studies have found that age differences in identifying both individual words and entire sentences can be significantly reduced by introducing a meaningful semantic context. As hearing loss occurs gradually over the adult lifespan (Morrell et al., 1996), older adults have increased opportunities to learn to use semantic context in order to compensate for age-related sensory declines and thereby exhibit relatively well-preserved understanding of meaningful passages.

What, then, was responsible for the sharp decline in listening comprehension in the oldest participants? One possibility is that by age 65 hearing loss may have reached 'critical values' such that compensation for reduced audibility using semantic context or other abilities was reduced, leading to greater impairments in listening comprehension for this group. Additionally, significant low-frequency hearing loss is evident for the oldest participants in our study and this may also have contributed to the progressive reduction in listening comprehension for this group.

An analogous argument concerning critical values could be made with respect to the perceptual effort hypothesis (McCoy et al., 2005). That is, hearing loss may mean that listeners must exert greater perceptual effort (with the extra effort required being an increasing function of the amount of loss), but listeners may be able to handle the greater effort requirement until it reaches some critical value. Recall that McCoy et al. found poorer memory for the initial word in three-word sequences in older adults with more impaired hearing, despite the fact that the words were intelligible for all participants, a result they attributed to increased perceptual effort during encoding. If the effects of such increased perceptual effort are not observed until some critical level is reached, this might explain why such "downstream" effects of encoding difficulty on listening comprehension were only observable in the oldest participants in the current study.

Finally, in addition to hearing loss, age-related perceptual and cognitive declines may also have contributed to the impaired listening comprehension abilities of the older participants in our study. Although verbal knowledge (e.g., vocabulary) remains relatively intact or even increases across most of the adult life span, processing speed declines markedly (Cerella \& Hale, 1994; Lima, Hale, \& Myerson, 1991), and the comprehension deficits observed in the oldest participants may have occurred because new information in the passages was presented before older participants had an opportunity to encode and integrate previous material (Salthouse, 1996). Similarly, working memory capacity decreases with age (Myerson, Hale, Rhee, \& Jenkins, 1999; Salthouse, 1994), and the observed comprehension deficits may reflect the fact that older adults are less able to remember what has just been said while simultaneously processing new information.

From a broader perspective, the picture that emerges from the present results is that listening comprehension joins a highly select group of abilities that show relative stability across most of the adult lifespan (Park \& Schwartz, 2000). The critical challenge for future 
investigations of listening comprehension will be twofold. First, it will be essential to identify both the perceptual and cognitive mechanisms mediating listening comprehension. To the extent that one or more of these abilities are amenable to training, such findings would allow for rehabilitation procedures specifically targeted at maintaining this critical ability, even for those over age 65 . Second, investigations aimed at specifying and training potential compensatory mechanisms used to maintain listening comprehension may help preserve people's ability to understand spoken messages even after age 65. Although such proposals remain speculative at this point, they illustrate the importance of identifying specific cognitive and perceptual abilities that mediate changes in listening comprehension as well as compensatory abilities that might mitigate the effects of age.

\section{Acknowledgments}

This work was supported by the National Institute on Aging and the Gustavus and Louise Pfeiffer Foundation. The authors would like to thank Denise Rose for her contribution to data collection.

\section{References}

American National Standards Institute. American national standard methods for the calculation of the speech intelligibility index. New York: Acoustical Society of America; 1997. ANSI S3.5-1997 Revision of ANSI S3.5-1969 (R1986).

Brandt J, Spencer M, Folstein M. The telephone interview for cognitive status. Neuropsychiatry, Neuropsychology, and Behavioral Neurology. 1988; 1:111-117.

Brown, JI.; Carlsen, GR.; Carstens, L. Brown-Carlsen-Carstens Listening Comprehension Test. New York: Harcourt, Brace and World; 1995.

Cerella J, Hale S. The rise and fall in information-processing rates over the life span. Acta Psychologica. 1994; 86:109-197. [PubMed: 7976466]

Ching TYC, Dillon H, Byrne D. Speech Recognition Of Hearing-Impaired Listeners - Predictions From Audibility and the Limited Role Of High-Frequency Amplification. Journal of the Acoustical Society of America. 1998; 103:1128-1140. [PubMed: 9479766]

Claman, C., editor. 10 real SATs. New York, NY: College Board; 1997.

Committee on Hearing and Bioacoustics, Working Group on Speech Understanding and Aging. Speech understanding and aging. Journal of the Acoustical Society of America. 1988; 83:859-895. [PubMed: 3281988]

Cruickshanks KJ, Wiley TL, Tweed TS, et al. Prevalence of hearing loss in older adults in Beaver Dam, Wisconsin. The epidemiology of hearing loss study. American Journal of Epidemiology. 1998; 148:879-886. [PubMed: 9801018]

de Jager CA, Budge MM, Clarke R. Utility of TICS-M for the assessment of cognitive function in older adults. International Journal of Geriatric Psychiatry. 2003; 18:318-324. [PubMed: 12673608]

Gates GA, Cooper JC, Kannel WB, et al. Hearing in the elderly: The Framingham cohort, 1983-1985. I. Basic audiometric test results. Ear and Hearing. 1990; 4:247-256. [PubMed: 2210098]

Hogan CA, Turner CW. High-Frequency Audibility - Benefits For Hearing-Impaired Listeners. Journal of the Acoustical Society of America. 1998; 104:432-441. [PubMed: 9670535]

Hornsby BW, Ricketts TA. The effects of hearing loss on the contribution of high- and low-frequency speech information to speech understanding. II. Sloping hearing loss. The Journal of the Acoustical Society of America. 2006; 119(3):1752-1763. [PubMed: 16583917]

Humes LE. Speech understanding in the Elderly. Journal of the American Academy of Audiology. 1996; 7:161-167. [PubMed: 8780988]

Hutchinson KM. Influence of sentence context on speech perception in young and older adults. Journal of Gerontology. 1989; 44:36-44.

Jerger J. Audiological findings in aging. Advances in Otology, Rhinology and Laryngology. 1973; 20:115-124.

Ear Hear. Author manuscript; available in PMC 2012 November 1. 
Kaufman AS, Horn JL. Age changes on tests of fluid and crystallized ability for women and men on the Kaufman Adolescent and Adult Intelligence Test (KAIT) at ages 17-94 years. Archives of Clinical Neuropsychology. 1996; 11:97-121. [PubMed: 14588911]

Lima SD, Hale S, Myerson J. How general is general slowing? Evidence from the lexical domain. Psychology and aging. 1991; 6(3):416-425. [PubMed: 1930758]

McCoy SL, Tun PA, Cox LC, et al. Hearing loss and perceptual effort: downstream effects on older adults' memory for speech. Quarterly Journal of Experimental Psychology A. 2005; 58:22-33.

Morrell CH, Gordon-Salant S, Pearson JD, et al. Age- and gender-specific reference ranges for hearing level and longitudinal changes in hearing level. Journal of the Acoustical Society of America. 1996; 100:1949-1967. [PubMed: 8865630]

Myerson J, Hale S, Rhee SH, Jenkins L. Selective interference with verbal and spatial working memory in young and older adults. Journals of Gerontology Series B-Psychological Sciences \& Social Sciences. 1999; 54:P161-P164.

Nittrouer S, Boothroyd A. Context effects in phoneme and word recognition by young children and older adults. Journal of the Acoustical Society of America. 1990; 87:2705-2715. [PubMed: 2373804]

Obler LK, Nicholas M, Albert ML, et al. On comprehension across the adult lifespan. Cortex. 1985; 21:273-280. [PubMed: 4028743]

Park, D.; Schwartz, N. Cognitive aging: A Primer. Oxfordshire; England: 2000.

Rajan R, Cainer KE. Ageing without hearing loss or cognitive impairment causes a decrease in speech intelligibility only in informational maskers. Neuroscience. 2008; 154:784-795. [PubMed: 18485606]

Salthouse TA. Aging associations: Influence of speed on adult age differences in associative learning. Journal of Experimental Psychology: Learning, Memory, \& Cognition. 1994; 20:1486-1503.

Salthouse TA. The processing-speed theory of adult age differences in cognition. Psychological Review. 1996; 103:403-428. [PubMed: 8759042]

Schneider BA, Pichora-Fuller MK. Age-related changes in temporal processing: Implications for speech perception. Seminars in Hearing. 2001; 22:227-240.

Schneider BA, Daneman M, Murphy DR, et al. Listening to discourse in distracting settings: The effects of aging. Psychology and Aging. 2000; 15:110-125. [PubMed: 10755294]

Sommers MS, Danielson SM. Inhibitory processes and spoken word recognition in young and older adults: the interaction of lexical competition and semantic context. Psychology and Aging. 1999; 14:458-472. [PubMed: 10509700]

Studebaker GA, Pavlovic CV, Sherbecoe RL. A frequency importance function for continuous discourse. Journal of the Acoustical Society of America. 1987; 81:1130-1138. [PubMed: 3571730]

Tye-Murray N, Sommers M, Spehar B, et al. Auditory-visual discourse comprehension by older and young adults in favorable and unfavorable conditions. International Journal of Audiolology. 2008; 47 2:S31-33.

Wechsler, D. Administration and scoring manual: WAIS-III. San Antonio, TX: Harcourt Brace \& Co; 1997. 


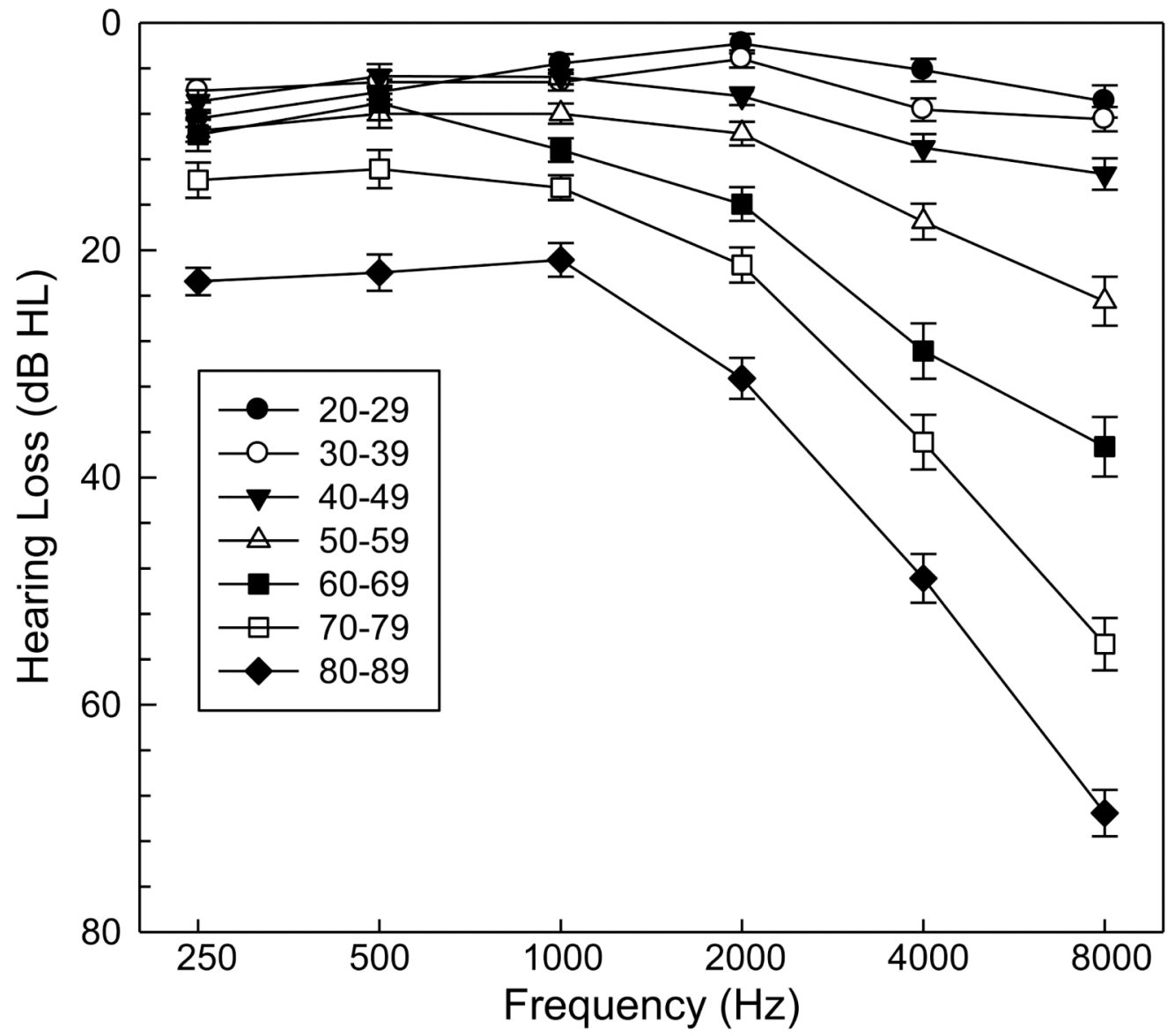

Figure 1.

Mean hearing loss (dB HL) in the best ear for participants in each of seven age groups plotted as a function of test frequency. 

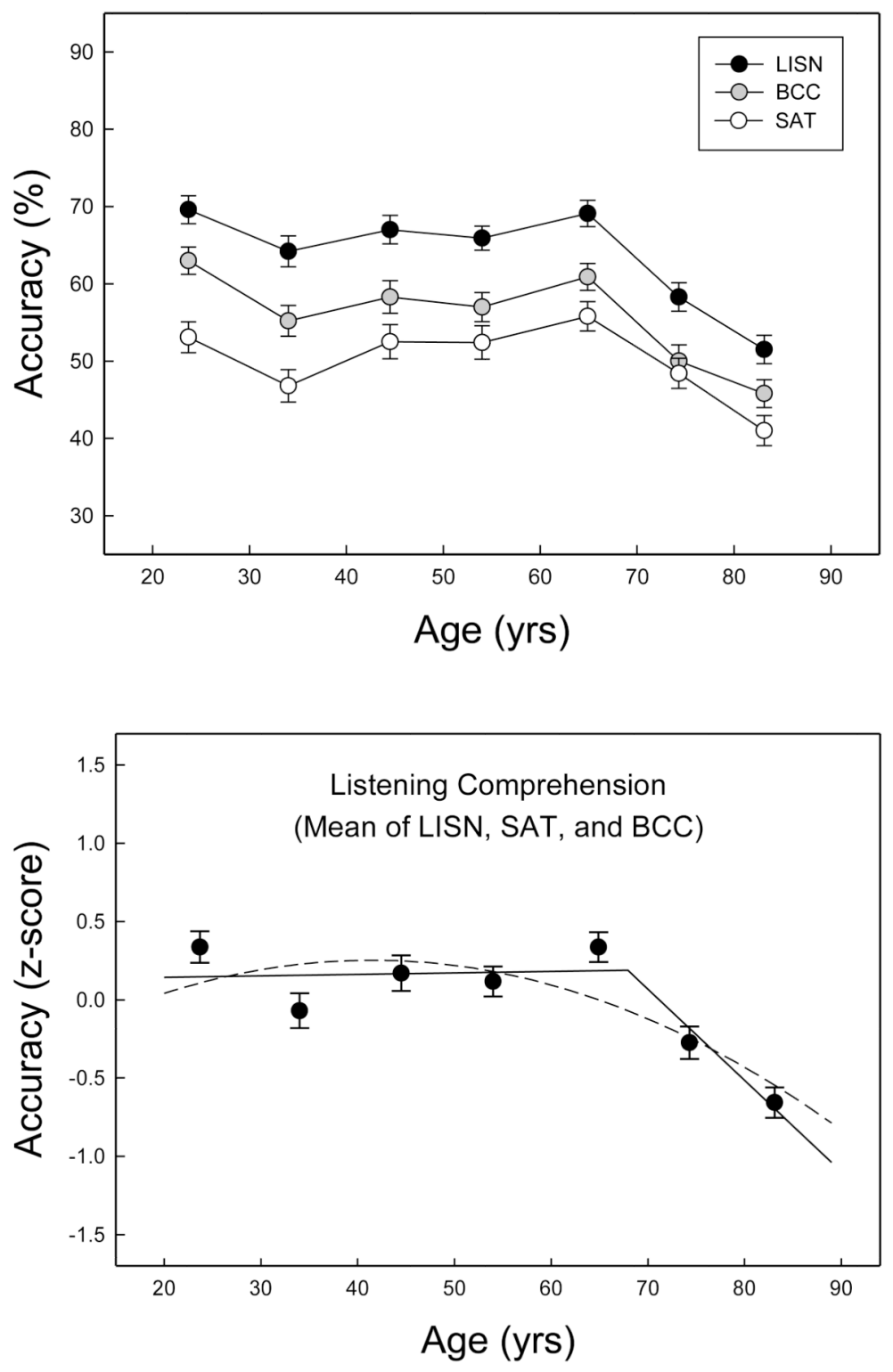

Figure 2.

(top) Mean percent correct for the LISN, SAT, and BCC measures of comprehension plotted as a function of age group. Error bars represent standard errors of the mean. (bottom)

Piecewise (solid line) and polynomial (dashed line) fits to mean (z score) listening comprehension performance across the three measures (BCC, SAT, and LISN) as a function of age. 


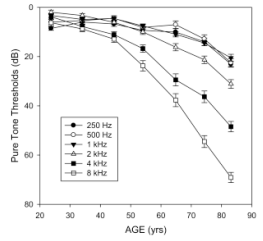

Figure 3.

Mean hearing loss (dB HL) in the best ear at different test frequencies plotted as a function of age. 


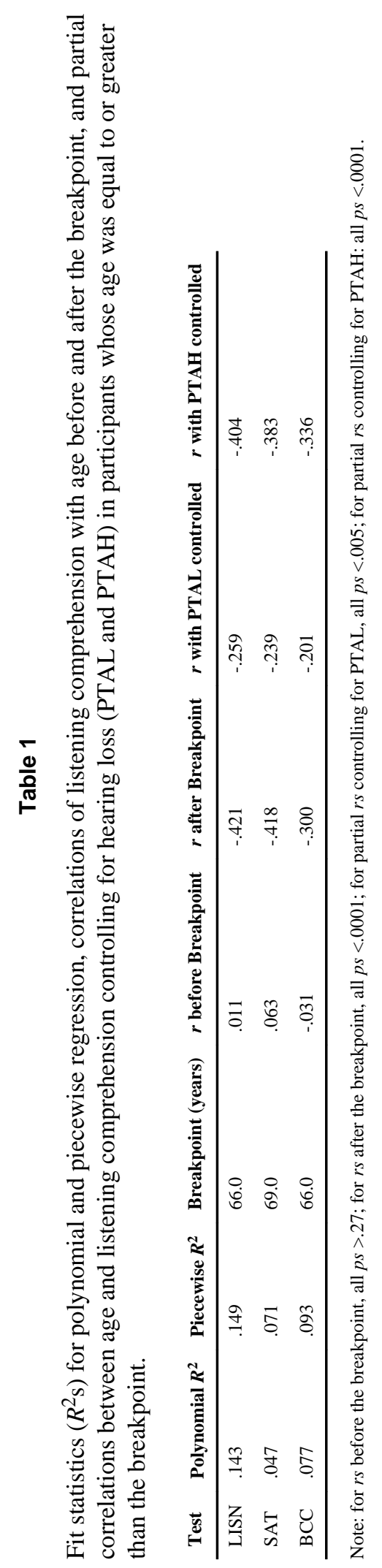

Ear Hear. Author manuscript; available in PMC 2012 November 1. 